Article

\title{
Characterization and Function of Two Short Peptidoglycan Recognition Proteins Involved in the Immunity of Bactrocera dorsalis (Hendel)
}

\author{
Dong Wei ${ }^{1,2}$, Yu-Wei Liu ${ }^{1,2}$, Ying-Xin Zhang ${ }^{1,2}$ and Jin-Jun Wang ${ }^{1,2, *}$ \\ 1 Chongqing Key Laboratory of Entomology and Pest Control Engineering, College of Plant Protection, \\ Southwest University, Chongqing 400716, China; dong_wei1988@yahoo.com (D.W.); \\ yuwei1994@yahoo.com (Y.-W.L.); zyxin1995@163.com (Y.-X.Z.) \\ 2 International Joint Laboratory of China-Belgium on Sustainable Crop Pest Control, State Cultivation Base of \\ Crop Stress Biology for Southern Mountainous Land, Academy of Agricultural Sciences, \\ Southwest University, Chongqing 400715, China \\ * Correspondence: wangjinjun@swu.edu.cn; Tel.: +86-23-6825-0255 or +86-23-6825-1269
}

Received: 19 February 2019; Accepted: 15 March 2019; Published: 19 March 2019

check for updates

\begin{abstract}
Peptidoglycans (PGNs) are major bacterial components recognized by the immune systems of insects and mammals. PGN recognition proteins (PGRPs) are widely distributed and highly conserved in vertebrates and invertebrates. PGRPs are a family of pattern recognition receptors that recognize peptidoglycan and regulate immune responses. In this study, we cloned two PGRP genes (BdPGRP-SA and BdPGRP-SD) from Bactrocera dorsalis (Hendel), which encode 192 and 196 amino acid residues, respectively. Both genes were highly expressed in adults, especially in the fat body and midgut. These two genes were up-regulated when challenged by the immune triggers, PGN-EB (Escherichia coli O111:B4) and PGN-SA (Staphylococcus aureus). The suppression of transcriptional expression of either gene by RNA interference (RNAi) resulted in increased sensitivities to Gram-negative E. coli and Gram-positive S. aureus PGNs. Suppression of BdPGRP-SA and $-S D$ expression by RNAi resulted in weak expressions of four antimicrobial peptides (AMPs) upon injected with E. coli or S. aureus. BdPGRP-SA and $-S D$ are involved in recognizing both Gram-negative and Gram-positive bacteria independently to activate the downstream AMP's response to bacterial infection.
\end{abstract}

Keywords: oriental fruit fly; peptidoglycan; peptidoglycan recognition protein; PGRP; antimicrobial peptide; innate immune

\section{Introduction}

Insects have an evolutionary conserved innate immune system that protects against pathogen infection [1,2]. The humoral immune reaction is involved in the production of antimicrobial peptides (AMPs), which are mainly synthesized in the fat body and then released into the hemolymph [3]. The expression of AMP genes is regulated by the Toll and immune deficiency (Imd) signal transduction pathways. The Toll pathway is activated by Gram-positive bacteria and fungi, while the Imd pathway is triggered by Gram-negative bacteria [4,5]. Microbial recognition represents the first step of the immune response. For pathogen detection, innate immunity functions with receptors that recognize the conserved motifs of pathogens (peptidoglycan $=$ PGN) but that are absent in the host [6]. PGN is recognized by conserved host PGN recognition proteins (PGRPs). All Gram-negative and a subset of Gram-positive bacteria (e.g. Bacillus) possess a meso-diaminopimelic acid (DAP) residue at the third position of the peptide bridge, whereas Gram-positive bacteria possess a lysine (Lys) [7-10]. 
Drosophila can discriminate between bacteria containing either Lys-type PGN (Lys-PGN) and DAP-type PGN (DAP-PGN) to elicit distinct antimicrobial responses via the selective activation of the Toll and Imd pathways, respectively [7]. Lys-PGN Gram-positive bacteria can be recognized by the secreted PGRP-SA, which activates the Toll pathway to stimulate the expression of antimicrobial peptide genes (e.g., Drosomycin) via the NF-kB members Dif and Dorsal [11,12]. In contrast, DAP-PGN Gram-positive bacteria trigger the Imd pathway through the NF- $\mathrm{kB}$ transcription factor Relish. For example, PGRP-SD acts upstream and activates the Imd pathway in Drosophila [13]. In insects, PGRPs are classified into short (PGRP-S) and long (PGRP-L) types according to their size. In D. melanogaster, there are six short PGRPs: PGRP-SA, $-S B,-S C 1 A,-S C 1 B,-S C 2$, and $-S D$ [14]. The short PGRPS are extracellular proteins prefixed with signal peptides, widely present in the hemolymph, cuticle, and fat body cells, and sometimes in epidermal cells of gut and hemocytes [10,15].

In Drosophila, some PGRPs function as enzymatic PGRPs (e.g., PGRP-SB and -SC), by removing the peptide from the glycan chains and cleaving it into non-immunized fragments $[16,17]$. Non-catalytic PGRP lost enzymatic activity but retained the ability to bind peptidoglycans, mediating microbial ligand-dependent downstream signaling [18]. For example, PGRP-SA activates the Toll pathway in response to Gram-positive bacterial infection in Drosophila $[19,20]$. The PGRPs independent functions in two pathways were also observed in B. mori. For example PGRP-L6, has a crucial role in Imd pathway activation [21], while PGRP-S5 negatively regulates AMP's production in an amidase-dependent fashion via the Imd pathway [22]. PGRP-SD can bind Gram-positive bacteria to activate the Toll pathway [20,23], and also act as a recognition protein that is required upstream of the Imd pathway for defense against Gram-negative bacteria $[24,25]$. The functions of PGRP-SA and -SD are complex and important in insect response to bacterial infection.

The oriental fruit fly, Bactrocera dorsalis (Hendel), attacks a variety of commercial fruits and vegetables [26]. To date, no studies have documented the PGRPs and their functions in the B. dorsalis immune system. Here, we cloned two short PGRPs (PGRP-SA and PGRP-SD) in B. dorsalis. Their expressions in different developmental stages and adult tissues were analyzed using RT-qPCR. Their functions were further investigated using RNA interference (RNAi). This study provided some basic information on immunity in $B$. dorsalis.

\section{Materials and Methods}

\subsection{Insects}

The laboratory strain of $B$. dorsalis was originally obtained from Haikou, Hainan province, China, in 2008, and was reared at $27.5 \pm 0.5^{\circ} \mathrm{C}$ with a relative humidity of $70 \pm 5 \%$ under a $14: 10 \mathrm{~h}(\mathrm{~L}: \mathrm{D})$ photoperiod. Newly laid eggs were transferred to an artificial diet as described previously $[27,28]$.

\subsection{Total RNA Extraction and First Strand cDNA Synthesis}

The total RNA was extracted using TRIzol reagent (Invitrogen, Carlsbad, CA, USA) from B. dorsalis female adults following manufacturer instructions. RNA was quantified with a NanoDrop One (Thermo Fisher Scientific, Madison, WI, USA), and the quality was evaluated via the absorbance ratio of optical density $\mathrm{OD}_{260 / 280}$ and $\mathrm{OD}_{260 / 230}$. To eliminate genomic DNA, the total RNA samples were treated with RQ1 DNaseI (Promega, Madison, WI, USA). Then first-strand cDNA was synthesized using PrimeScript ${ }^{\circledR}$ RT reagent Kit (Takara, Dalian, China) according to the standard manufacturer protocol. Briefly, the reaction volume was $10 \mu \mathrm{L}$, which contained $\approx 500 \mathrm{ng}$ of total RNA, 200 pmol of random hexamers, $2 \mu \mathrm{L}$ of reverse transcription buffer, $0.5 \mu \mathrm{L}$ of reverse transcriptional enzyme mix, and additional RNase-free $\mathrm{H}_{2} \mathrm{O}$ to a final volume of $10 \mu \mathrm{L}$. The reverse transcription reaction was performed on a C1000TM Thermal Cycler (Bio-Rad, Hercules, CA, USA) at $37^{\circ} \mathrm{C}$ for $15 \mathrm{~min}$ followed by $85^{\circ} \mathrm{C}$ for $5 \mathrm{~s}$. 


\subsection{Cloning of BdPGRP-SA and BdPGRP-SD}

The fragments of two genes (BdPGRP-SA and BdPGRP-SD) were obtained from the transcriptomes of $B$. dorsalis [29]. The deduced amino acid sequences of selected PGRPs genes were predicted using the online open reading frame (ORF) Finder (http:/ / www.ncbi.nlm.nih.gov/gorf/orfig.cgi). Two pairs of gene-specific primers were designed using DNAMAN 7.0 (Lynnon Biosoft, QC, Canada) to amplify the ORFs (Table S1). PCR was carried out in a $25 \mu \mathrm{L}$ reaction volume using high fidelity DNA polymerase PrimerSTAR kit (TaKaRa) with the following procedure: $98^{\circ} \mathrm{C}$ for $2 \mathrm{~min}$, and then 35 cycles of $95^{\circ} \mathrm{C}$ for $30 \mathrm{~s}, 60^{\circ} \mathrm{C}$ for $30 \mathrm{~s}$, and $72{ }^{\circ} \mathrm{C}$ for $1 \mathrm{~min}$, followed by a final step of $72{ }^{\circ} \mathrm{C}$ for $10 \mathrm{~min}$. The PCR products were checked on a $1.0 \%$ gel after electrophoresis and then purified using a gel extraction kit (Takara). The purified fragments were cloned into pGEMT Easy Vector (Promega) and transformed into DH5 $\alpha$ competent cells (Biomed, Beijing, China). We used Luria-Bertani agar plates containing $0.1 \%$ ampicillin to select the positive transformants for sequencing (BGI, Beijing, China).

\subsection{Characterization, Sequence Alignment, and Phylogenetic Analysis}

The amino acid sequences of both PGRPs were predicted using Primer Premier 5.0 software (Premier Biosoft, Palo Alto, CA, USA). The potential PGRP protein sequences were further analyzed for the presence of putative functional domains using InterProScan 5 (https:/ /www.ebi.ac.uk/interpro), for signal peptides prediction using SignalP 4.1 Server (http:/ / www.cbs.dtu.dk/services/SignalP), and for transmembrane domains prediction using TMHMM Server 2.0 (http:/ / www.cbs.dtu.dk/services/ TMHMM-2.0). The homologous sequences from other insect species, including D. melanogaster, Tribolium castaneum, Culex quinquefasciatus, Anopheles gambiae, and B. mori were obtained from the NCBI database via BLAST. The sequence alignment of BdPGRP-SA and BdPGRP-SD with the homologs from other insects were performed using Clustal omega and visualized using Jalview 2.0 software [30]. The phylogenetic tree of amino acid sequences of the PGRPs was constructed using a neighbor-joining method with MEGA 5.0 with 1000 bootstrap replicates [31].

\subsection{Quantitative Real-Time Polymerase Chain Reaction ( $q R T-P C R$ )}

To determine the expression profiles of BdPGRP-SA and -SD in different developmental stages and tissues, we sampled eggs; 1-, 3-, 5-, and 8-day-old larvae; 1-, 3-, 5-, and 7-day-old pupae; and $1-, 3-, 5-$, and 7-day-old adults, as well as various body parts or tissues from 5-day-old adults. Several individuals were pooled together into one replicate. The adult central nervous system, midgut, fat body, Malpighian tubules, hindgut, ovary, and testis were dissected and used for total RNA isolation as above. After preparation of templates, a CFX384 Optics Module (Bio-Rad, Singapore) was used for qRT-PCR. Each $10 \mu \mathrm{L}$ of PCR reaction mixture consisted of $5 \mu \mathrm{L}$ of Novostar-SYBR Supermix (Novoprotein, Shanghai, China), $3.5 \mu \mathrm{L}$ of nuclease-free water, $0.5 \mu \mathrm{L}$ of cDNA $(\approx 500 \mathrm{ng} / \mu \mathrm{L})$, and $0.5 \mu \mathrm{L}$ each of forward and reverse primers $(10 \mu \mathrm{M})$. The qPCR condition was as follows: initial denaturation at $95{ }^{\circ} \mathrm{C}$ for $2 \mathrm{~min}$, followed by 40 cycles of $95{ }^{\circ} \mathrm{C}$ for $15 \mathrm{~s}$ and $60{ }^{\circ} \mathrm{C}$ for $30 \mathrm{~s}$. At the end of the procedure, a melting curve analysis from 60 to $95^{\circ} \mathrm{C}$ was recorded to ensure the specificity and consistency of all generated products. Alpha-tubulin (GenBank: GU269902) was used as an internal reference gene based on previous evaluations [32]. A standard curve was established for each set of primers to validate their specificity. All of the experiments were performed in three biological replicates, and the data were analyzed using the comparative $2^{-\Delta \Delta C T}$ method [33].

\subsection{Immune Triggers Induction}

The peptidoglycan of PGN-EB from the Gram-negative E. coli O111:B4 (Invivogen, San Diego, CA, USA) and PGN-SA from the Gram-positive Staphylococcus aureus (Invivogen) were used to test the response of BdPGRP-SA and BdPGRP-SD to microbial pathogen infection in B. dorsalis. PGN-EB, purified from E. coli, and PGN-SA, purified from the S. aureus, were diluted in $1 \times \mathrm{PBS}(\mathrm{pH} 7.2)$ to a final concentration of $100 \mathrm{ng} / \mu \mathrm{L}$. For stimulus and transcriptional analysis, 5 -d-old adult $B$. dorsalis 
were injected with $200 \mathrm{~nL}$ of PGN-SA and PGN-EB solutions, respectively. Flies injected with $200 \mathrm{~nL}$ PBS only were the negative control. Treated flies were collected at 3, 6, 9, 12, and $24 \mathrm{~h}$ after injection. All of the samples were collected for total RNA extraction immediately as described above for gene expression determination using qRT-PCR. Three replicates at each time point were performed.

\subsection{RNA Interference (RNAi)}

RNAi was used to explore the potential function of BdPGRP-SA and BdPGRP-SD in B. dorsalis. The unique nucleotide regions of $B d P G R P-S A$ and $-S D$ were selected for dsRNA synthesis using gene-specific primers (Table S1). DsRNAs of BdPGRP-SA and -SD were synthesized using a TranscriptAid T7 High Yield Transcription Kit (Thermo Scientific, Vilnius, Lithuania). Agarose gel electrophoresis was used to measure the size and quality of dsRNA and the NanoDrop One spectrophotometer was used to test the dsRNA concentration. The dsRNA of GFP was used as the negative control [34]. Approximately $1.2 \mu \mathrm{g}$ of dsPGRPS or dsGFP was injected into 5-day-old $B$. dorsalis adults using a Nanoject II Auto-Nanoliter Injector (Drummond Scientific, Broomall, PA, USA). Tested flies (male to female ratio 1:1) were collected at $24 \mathrm{~h}$ after injection and total RNAs were isolated for gene silencing efficiency determination. Another batch of dsRNA-injected flies were collected and injected with living E. coli (Gram-negative) and S. aureus (Gram-positive) after $24 \mathrm{~h}$. Each insect was injected with $200 \mathrm{~nL}$ E. coli solution with an $\mathrm{OD}_{600}$ value of 2.0 and S. aureus with an $\mathrm{OD}_{600}$ value of 1.0 . Control flies were injected with $200 \mathrm{~nL}$ of PBS solution. In each group, a total of 60 flies were injected and their survival rates were recorded for at least 5 days.

We also evaluated the AMPs gene expression after gene silencing followed by bacterial infection. The alive bacteria were inactivated by incubating at $65{ }^{\circ} \mathrm{C}$ for $10 \mathrm{~min}$. Flies were injected with inactivated E. coli and S. aureus, at $24 \mathrm{~h}$ after dsRNA injection. The control flies were injected with $200 \mathrm{~nL}$ of PBS. The flies were collected at $24 \mathrm{~h}$ after inactive pathogens injection for transcriptional analysis. The relative expression level of target genes and four downstream AMPs genes, attacin- $A$ (GenBank accession number: KY038167), cecropin-2 and defensin (GenBank accession number: KX510001 and KX510002) [35], and diptericin (GenBank accession number of KJ488999) [36], were selected for gene expression determination by RT-qPCR.

\subsection{Statistical Analysis}

All of the experiments were conducted with three biological replications on the same day. The differences of gene expression levels were analyzed using one-way analysis of variance (ANOVA), followed by Tukey's honestly significant difference (HSD) multiple comparison test using SPSS ver. 19.0 software (IBM, Chicago, IL, USA). The differences between pathogen treatment and silencing efficiency were determined by the independent sample Student's $t$ test $(p<0.05)$.

\section{Results}

\subsection{Sequence Analysis, Alignments, and Phylogenetic Trees}

The cDNA sequences of BdPGRP-SA and BdPGRP-SD were cloned from adult female B. dorsalis. The full-length cDNA sequence of BdPGRP-SA and BdPGRP-SD contained an open reading frame of $579 \mathrm{bp}$ and $591 \mathrm{bp}$, encoding 192 and 196 amino acid residues, respectively. Both genes were uploaded to NCBI GenBank with accession numbers of MK392505 and MK392506. Based on the conserved domain search result on NCBI, BdPGRP-SA and BdPGRP-SD were both expected to be members of the PGRP superfamily. According to SignalP 4.0, predicted signal peptides for PGRP-SA and PGRP-SD were 21 and 21 amino acids, respectively, but no transmembrane domain was predicted (Figure 1A). Alignment was carried out using the Clustal Omega program and visualized using Jalview software 2.9 (http:/ / www.mybiosoftware.com/jalview-2-6-1-multiple-alignment-editor.html) (Figure 1B). Only BdPGRP-SD has an Arg105 amino acid residue, which was required for the recognition of DAP-PGN. The two conserved cysteines were determined in both sequences. Comparative analysis 
indicated that the amino acids of His57, Tyr91, Thr171, which are required for T7 lysozyme $\mathrm{Zn}^{2+}$ binding and amidase activity, were conserved in BdPGRP-SD (Figure 1B). The Trp86 was not in both sequences, indicating that one of the PGRPs was catalytic PGRPs.

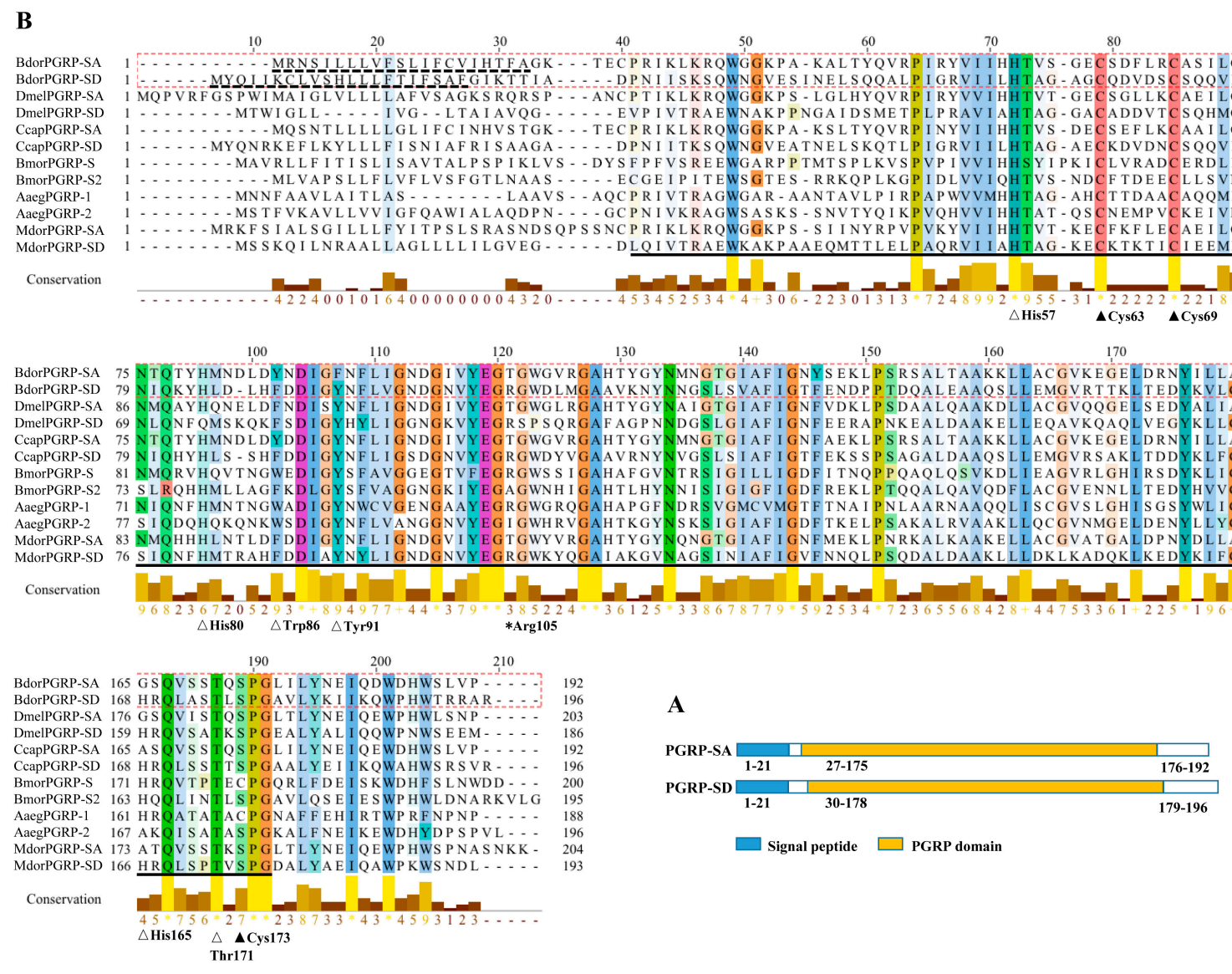

Figure 1. Structure of the signal peptide and conserved domain of two BdPGRPs (A), and multiple sequence alignment of PGRPs from insects (B). The blue bar and yellow bars in panel A indicate the signal peptide and conserved PGRP domain, respectively. The dashed line indicates the signal peptide amino acids, and the black line indicates the conserved PGRP domain region. The black triangles in panel B indicate the conserved cysteine residues, the clear triangles indicate the amino acid residues required for PGRP/amidase activity, and the asterisk indicates the amino acid responsible for the recognition of DAP-PGN. The heights of the yellow or brown bars below the aligned sequences represent the degree of similarity of the amino acids. Bdor, Dmel, Ccap, Bmor, Aaeg, and Mdom indicate the insects of Bactrocera dorsalis, Drosophila melanogaster, Ceratitis capitata, Bombyx mori, Aedes aegypti, and Musca domestica, respectively.

A phylogenetic tree was constructed using the neighbor-joining method to analyze the relationships among PGRP-SA and PGRP-SD proteins in B. dorsalis with other insect PGRPs. BdPGRP-SA and BdPGRP-SD clustered into two separate clades that are the same clades as their orthologues homologs in other species (Figure 2). BdPGRP-SA was closely related to the PGRP-SA in C. capitata and Bactrocera oleae. However, BdPGRP-SD was closely related to the PGRP-SD in B. oleae. 


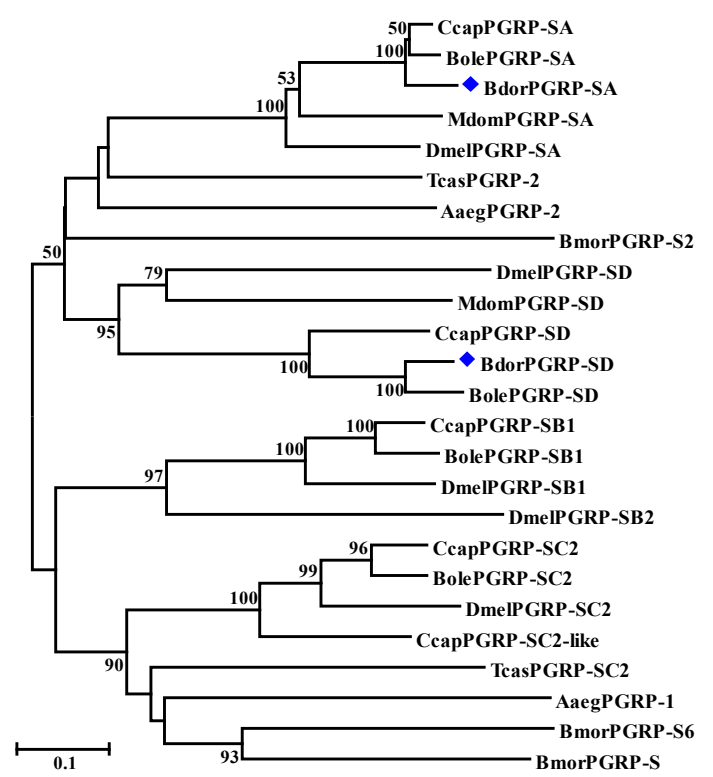

Figure 2. Phylogenetic analysis of the insect PGRPs. MEGA 5 was used to construct the phylogenetic tree using the neighbor-joining method with 1000 bootstrap replicates. All of the homologous PGRPs were retrieved from the NCBI. Insect species include Drosophila melanogaster (Dmel), Aedes aegypti (Aaeg), Bactrocera oleae (Bole), Ceratitis capitata (Ccap), Musca domestica (Mdom), and Tribolium castaneum (Tcas). Table S2 shows the amino acid sequences of the PGRPs used in the phylogenetic tree.

\subsection{Spatiotemporal Expression Analysis}

We determined the expression of BdPGRP-SA and -SD in the developmental stages and tissues of B. dorsalis using qRT-PCR. BdPGRP-SA was expressed in all of the developmental stages (Figure 3A). The expression of BdPGRP-SA increased during the larval development [37]. The expression of $B d P G R P-S D$ was significantly higher in adults (Figure 3B). Among the different tissues of adult flies, $B d P G R P-S A$ was highly expressed in fat body and hindgut (Figure 3C). BdPGRP-SD was highly expressed only in fat body, but had low expression in other tissues (Figure 3D).
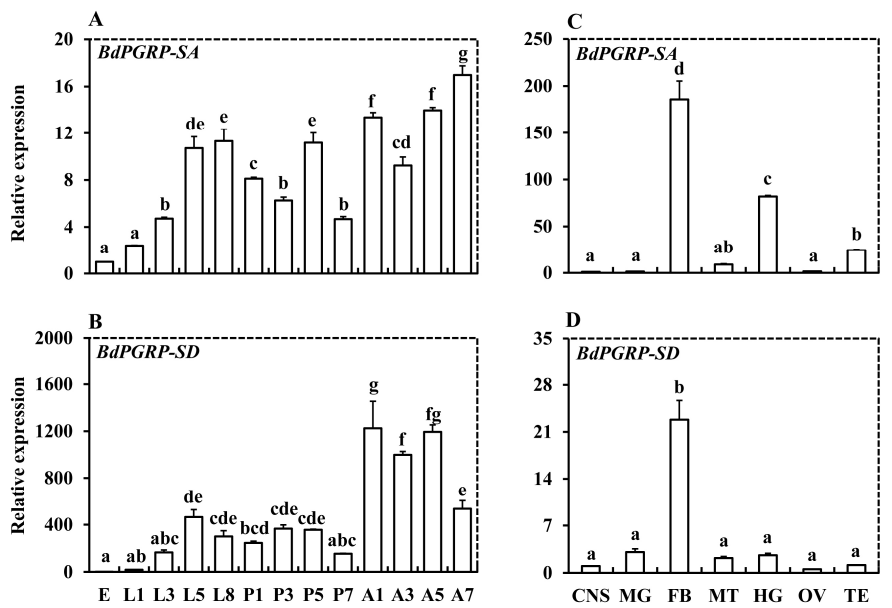

Figure 3. Gene expression profiles of $B d P G R P-S A$ and $B d P G R p-S D$ in the different developmental stages (A and B), and tissues of 5-day-old Bactrocera dorsalis adults (C and D). Egg (E); larvae on days 1, 3, 5, and 8 (L1, L3, L5, L8); pupae on days 1, 3, 5, and 7 (P1, P3, P5, P7); and adults on days 1, 3, 5, and 7 (A1, A3, A5, A7) of $B$. dorsalis were sampled for developmental stage expression analysis. Central nervous (CNS), midgut (MG), hindgut (HG), fat body (FB), Malpighian tubule (MT), ovary (OV), and testis (TE) in 5-day-old adults were collected for tissue expression determination. Different letters above the error bars indicate statistical differences $(p<0.05)$. 


\subsection{Expression Induced by Microbial Challenges}

To study the potential role of BdPGRP-SA and -SD in B. dorsalis immunity, 5-day-old adults were injected with the PGN-EB and PGN-SA. BdPGRP-SA and -SD were up-regulated to a peak at $12 \mathrm{~h}$ and $9 \mathrm{~h}$ respectively, and then decreased to a normal level at $24 \mathrm{~h}$ when challenged by PGN-EB (Figure 4A,B). When challenged by PGN-SA, the expression of BdPGRP-SA and BdPGRP-SD were up-regulated with a dynamic fluctuation. BdPPGRP-SA was up-regulated to be bi-modal with one peak at $3 \mathrm{~h}$ and a second at $12 \mathrm{~h}$ (Figure $4 \mathrm{C}$ ). While BdPGRP-SD showed a similar profile but with a first peak at $6 \mathrm{~h}$ and a second at $24 \mathrm{~h}$ (Figure $4 \mathrm{D})$.

A
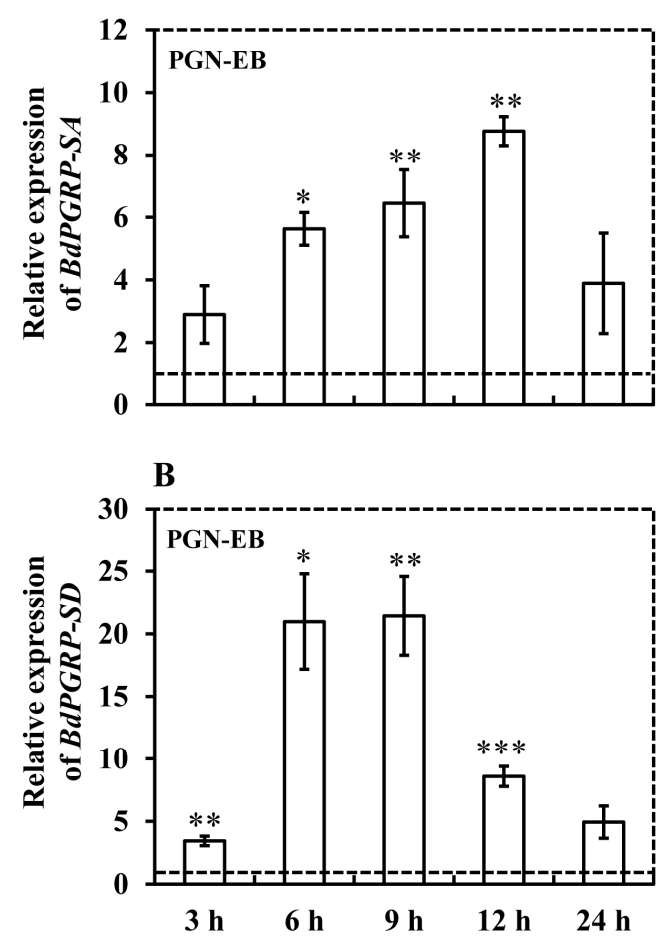

C

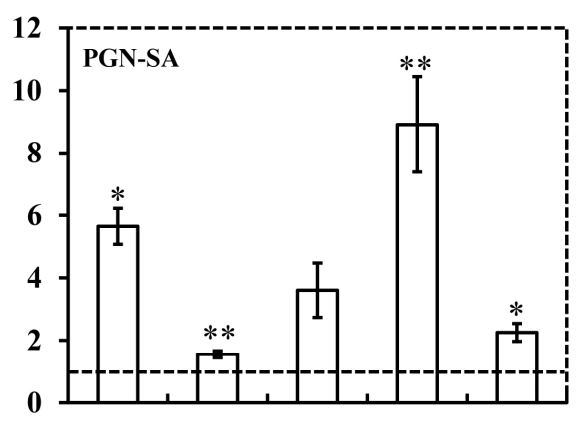

D

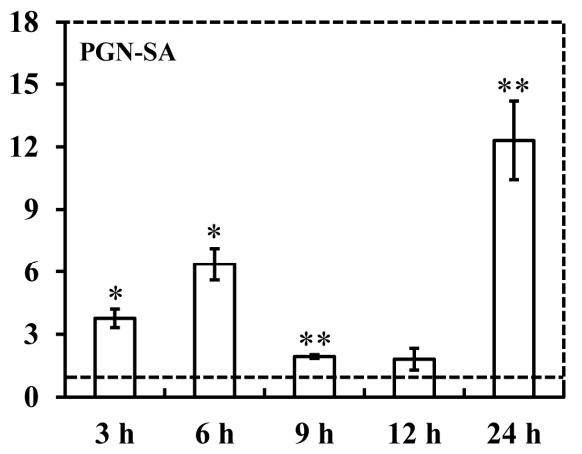

Figure 4. The expression of BdPGRP-SA and BdPGRp-SD in 5-day-old adults injected with peptidoglycan of PGN-EB and PGN-SA. PGN-EB and PGN-SA represents peptidoglycan from Escherichia coli 0111:B4 and Staphylococcus aureus, respectively. The control group was injected with an equal amount of PBS. Two flies (one male and one female) were collected and pooled at 3, 6, 9, 12, and $24 \mathrm{~h}$ post-pathogen injection. Significant differences were determined via the independent samples $t$-test ${ }^{*}$ indicates $p<0.05,{ }^{* *}$ indicates $p<0.01$, and ${ }^{* * *}$ indicates $p<0.001$ ).

\subsection{RNAi Bioassay}

At $24 \mathrm{~h}$ after injection of gene-specific dsRNA, the transcriptional expression of BdPGRP-SA and -SD decreased by $65.5 \%$ and $69.1 \%$, respectively (Figure 5A,B). When injected with E. coli and S. aureus, the dsBdPGRP-SA and -SD injected flies showed significantly lower survival rates within 5 days (Figure 5C,D). Survival at 5 days in the dsGFP groups was $88 \%$ when injected with E. coli. Survival of the dsBdPGRP-SA and -SD groups was significantly lower at $64 \%$ and $40 \%$, respectively. In the S. aureus injection group, the survival rate of dsGFP was $88 \%$, while the survival of dsBdPGRP-SA and $-S D$ were $28 \%$ and $59 \%$, respectively. BdPGRP-SD was more sensitive to E. coli and the BdPGRP-SA was more sensitive to $S$. aureus. 


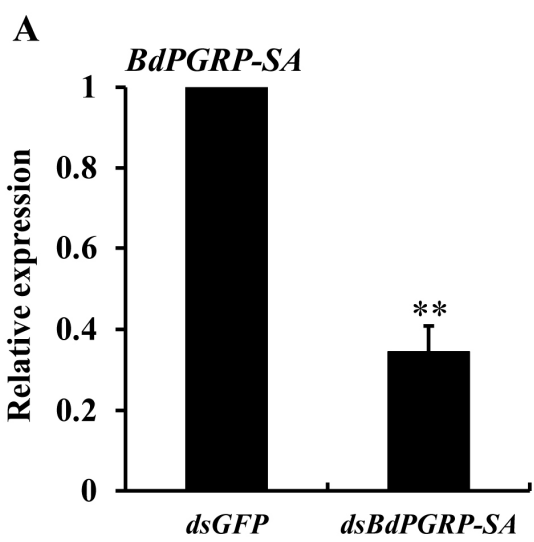

$\mathbf{B}$

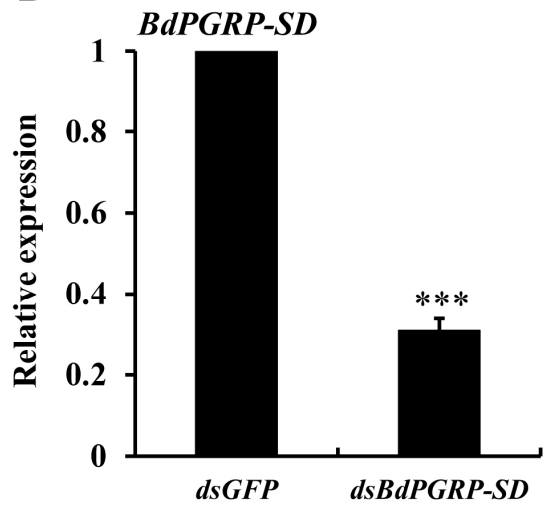

C

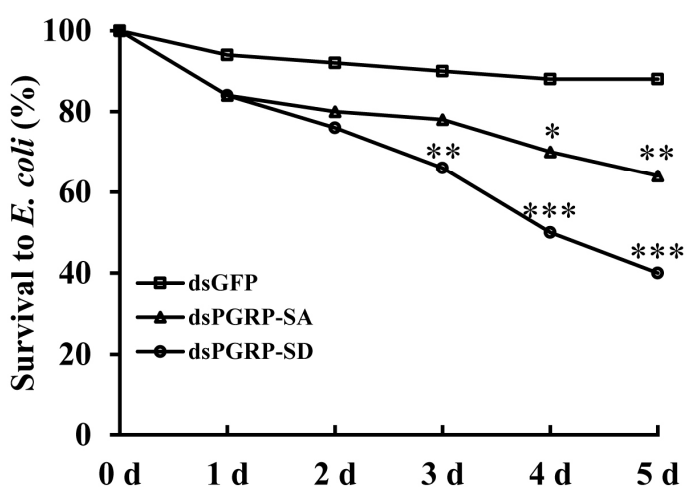

D

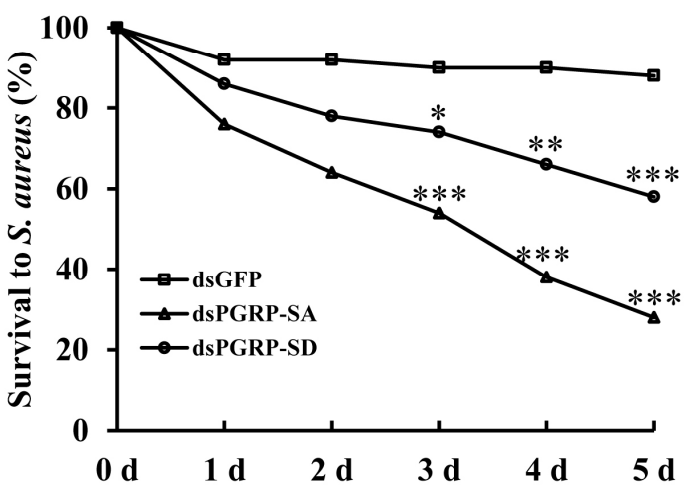

Figure 5. Relative transcriptional expression of $B d P G P R-S A$ and $B d P G R P-S D$ after gene-specific dsRNA injection (A and B), and the survival of flies after dsRNA injection followed by E. coli and S. aureus injection (C and D). The gene silencing efficiency was detected at $24 \mathrm{~h}$ after injection. Survival rates were calculated within 5 days after gene-specific dsRNA injection and E. coli and S. aureus $24 \mathrm{~h}$ later. Same amounts of dsGFP was injected as the negative control. Significant differences were determined by the independent samples $t$-test ( ${ }^{*}$ indicates $p<0.05$, ${ }^{* *}$ indicates $p<0.01$, and ${ }^{* * *}$ indicates $p<0.001$ ).

\subsection{Expression of Antimicrobial Peptides (AMPs)}

Flies injected for gene-specific suppression were collected for microbial injection at $24 \mathrm{~h}$. The transcription level of four AMPs genes, including attacin- $A$, cecropin-2, defensin, and diptericin, were determined using RT-qPCR. The gene expressions of the four AMPs were significantly up-regulated by Gram-negative and Gram-positive bacteria (Figure 6). In the E. coli treatment, the expressions of the four AMPs genes-attacin-A, cecropin-2, defensin, and diptericin-were induced to a high level when the $B d P G R P$-SA target gene was independently suppressed (Figure 6A). There was no difference between the BdPGRP-SA dsRNA injection group and the control. In the BdPGRP-SD dsRNA injection, three AMPs, including attacin- $A$, defensin, and diptericin, were not induced, and showed significantly lower expression than the negative control. This indicated that it was BdPGRP-SD, not BdPGRP-SA, that recognized the Gram-negative pathogen (i.e., E. coli) infection and responded by activating the expression of three AMPs. A similar trend of cecropin-2 expression also occurred in response to the E. coli infection. When flies were challenged by S. aureus, two downstream AMPs (cecropin-2 and diptericin) were expressed at a significantly lower level after BdPGRP-SA silencing (Figure 6B). This indicates that cecropin-2 and diptericin could be induced via the recognition of BdPGRP-SA against Gram-positive bacteria. When injected with S. aureus, cecropin-2 and defensin were expressed at a lower level in $B d P G R P-S D$ suppressed flies, indicating that $B d P G R P-S D$ was also involved in response to Gram-positive bacteria (Figure 6B). 

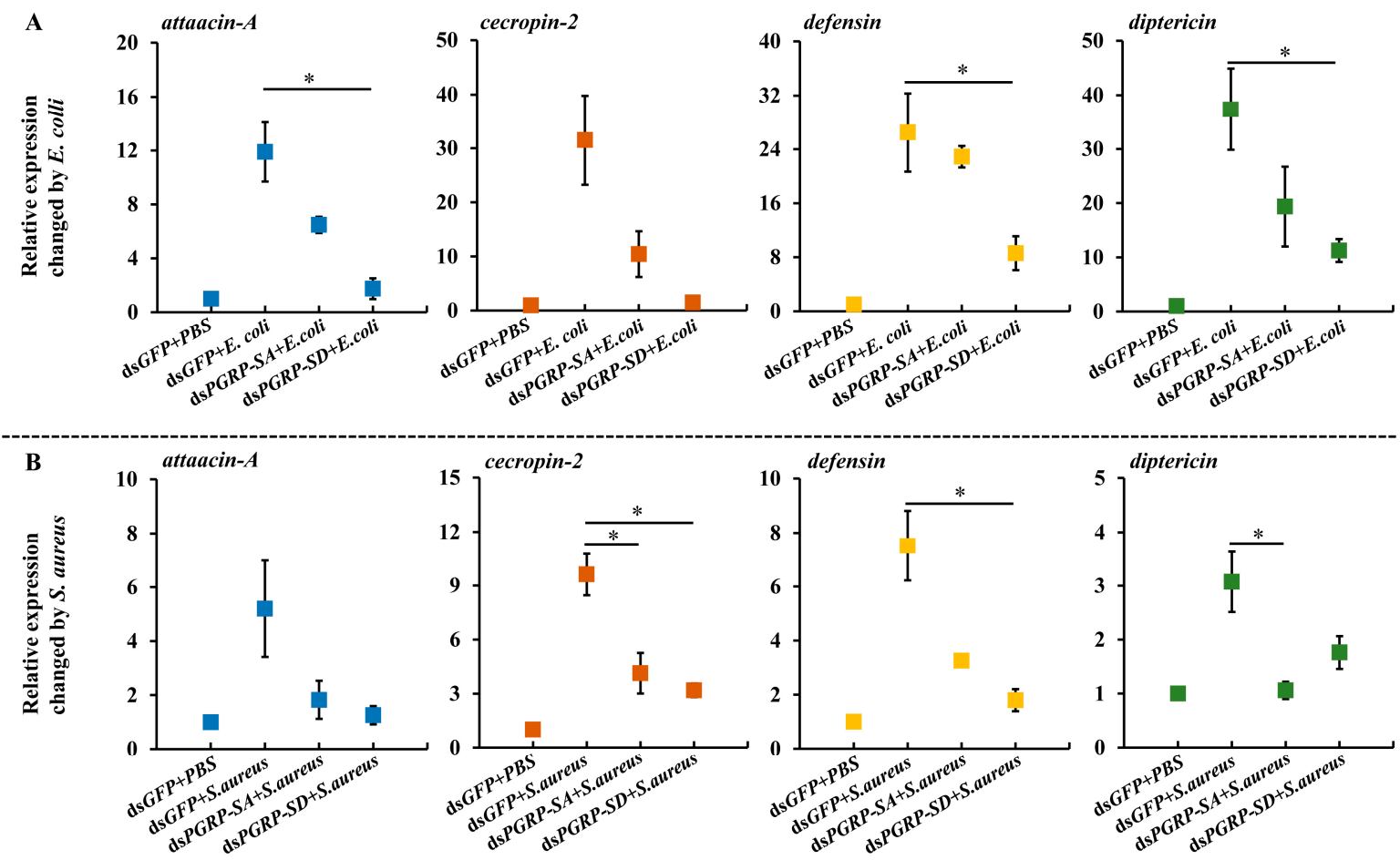

Figure 6. Relative expression level of antibacterial peptides attacin-A, cecropin-2, defensin, and diptercin after injection with dsRNA and injection with inactivated Escherichia coli (A) and Staphylococcus aureus (B). The data represent mean \pm SE. Significant differences determined by the independent samples $t$-test $\left({ }^{*}\right.$ indicates $\left.p<0.05\right)$.

\section{Discussion}

Insects rely on innate immunity for defense against microbial pathogens. Defenses include both the inducible AMPs and the activated prophenoloxidase cascades in the hemolymph [38]. In insects, peptidoglycan recognition proteins (PGRPs) play key roles in recognizing bacterial infection [7]. In this study, we cloned the transcripts of BdPGRP-SA and BdPGRP-SD found in B. dorsalis. The Arg amino acid residue was only found in BdPGRP-SD sequence and this is required for the recognition of DAP-PGN. His, Tyr, and Thr amino acids, required for T7 lysozyme $\mathrm{Zn}^{2+}$ binding and amidase activity, were partially conserved in two BdPGRP-Ss. For example, Tyr was replaced by Phe residue in BdPGRP-SA. The catalytic domain was associated with Gram-negative binding protein 1 (GNBP1) in activating the Toll receptor pathway [39]. Both genes may be involved in different pathways. In Drosophila, the PGRP-SA and -SD can discriminate different bacteria containing either Lys-PGN and DAP-PGN to elicit down-stream antimicrobial responses via activating the Toll and Imd pathways respectively [7]. In Antheraea pernyi, the conserved Tyr was also replaced by another amino acid, indicating a pattern recognition receptor in the immune system [40]. The amino acid Trp was also not conserved in B. dorsalis in the alignment, but it is conserved in mosquitoes, e.g., Aedes aegypti [41]. These findings indicate non-catalytic PGRPs produced by two genes in B. dorsalis. Similarly, in Drosophila, the homolog PGRP-SA and -SD are non-catalytic PGRPs [42]. Phylogenetic tree comparison of the BdPGRP-SA and -SD with the homologs from other insects showed that the both proteins PGRPs are closely located with that from B. oleae and M. domestica.

Insect PGRP-Ss are present in the hemolymph and cuticle. They are constitutively synthesized or induced mainly in the fat body cells and sometimes in epidermal cells in the gut, and to a lesser extent, in hemocytes [43,44]. In Drosophila, PGRP-SD is moderately expressed in the fat body and midgut of adult flies and highly expressed in the fat body of larvae [25]. In this study, BdPGRP-SA was highly expressed in both larvae and adults, and BdPGRP-SD was highly expressed in adults. The fat body is an important immune organ that responds to pathogen infection, so it is reasonable that both 
genes were highly expressed in this tissue. In Drosophila, PGRP-SA and GNBP1 are secreted into the hemolymph and combine into a complex that activates a proteolytic cascade that culminates in the cleavage of Spätzle and the Toll signaling pathway [11,19].

Insect PGRPs are involved in many important signal pathways in the innate immune system. In Drosophila, PGRP-SA and -SD recognize bacterial PGN and activate the Toll receptor $[19,20]$. In $B$. dorsalis, the expression of BdPGRP-SA was significantly increased after induction by PGN-EB and PGN-SA. This indicated that BdPGRP-SA can be induced by E. coli and S. aureus (Figure 4). The inducible expression of PGRPs has been observed in Armigeres subalbatus [45], A. aegypti [41], and Microplitis mediator [46]. The induction of BdPGRP-SA and -SD by PGN-EB was confirmed earlier [47]. PGRP-SD was previously considered to be a pattern recognition receptor (PRR) for Gram-positive bacteria, but subsequent biochemical and structural analysis revealed that PGRP-SD holds a peptidoglycan-binding groove characteristic of DAP-type recognition PGRPs. An in vitro binding study demonstrated that PGRP-SD binds DAP-PGN but not Lys-PGN [24]. PGRP-SA is required for triggering the Toll pathway whereas PGRP-SD is not essential but enhances signaling. Toll activation initiates a signal transduction cascade that results in the expression of AMPs [39].

In Drosophila, all of the flies died within 3 days after Gram-positive bacteria, S. aureus, Enterococcus faecalis, and Micrococcus luteus injection when PGRP-SA was mutated [11,20]. Similarly, PGRP-SD mutants were sensitive to $S$. aureus and S. pyogenes [20]. The survival phenotype could be rescued by injection of recombinant PGRP-SD before being challenged with S. aureus [23]. When combined with the PGRP-SA mutant, this mutation further impaired expression of the Toll pathway target genes [20]. In the present study, after infection with E. coli and $S$. aureus, the mortality rate of B. dorsalis adults injected with dsBdPGRP-SA increased by $24 \%$ and $60 \%$, respectively. This demonstrated that flies injected with dsBdPGRP-SA were more sensitive to $S$. aureus. The mortality of flies injected with dsBdPGRP-SD increased by $48 \%$ and $30 \%$ after E. coli and S. aureus infection, indicating a relatively higher sensibility to E. coli (Figure 5). The motilities of the two bacterial infections indicated that BdPGRP-SA was mainly involved in Gram-positive bacteria, possibly because of the replacement of the conserved Arg105 by a Thr. While BdPGRP-SD was involved in both Gram-negative and positive bacteria. A similar result was found in Drosophila where PGRP-SD mutants had increased susceptibility to some DAP-PGN bacteria species [12]. In Drosophila, PGRP-SD mutants had a reduced systemic Imd pathway activation and increased susceptibility to Gram-negative bacteria [25]. This revealed a new regulating Imd signaling pathway instead of the Toll pathways. The new functional mechanism was thereafter covered to enhance peptidoglycan-mediated activation of the Imd pathway by promoting peptidoglycan re-localization to the cell-surface receptor PGRP-LC [25].

Numerous AMPs have been identified, but their production pathways are usually unclear. When challenged by Erwinia carotovora or E. coli, the expression levels of AMPs transcripts remained at a high level in Drosophila [11,48]. Similarly, the transcription level of AMP genes, including attacin, cecropin, and lysozyme, were greatly up-regulated following the infection of E. coli and S. aureus, but they decreased when endogenous PGRP-SA was knocked down in A. pernyi [41]. Our data showed that E. coli induced a high expression of AMPs, but silencing of BdPGRP-SA did not result in low expression of the AMPs. When challenged by S. aureus, cecropin-2 and diptericin were inhibited in $d s B d P G R P-S A$ injected flies. This indicated a role of BdPGRP-SA in response to Gram-positive bacterial infection by activating downstream AMPs expression, e.g., cecropin-2 and diptericin. Similarly, when PGRP-SA was suppressed in $A$. pernyi, the expression of cecropin-B was not induced, i.e., exactly one fifth, after challenge by E. coli [41]. Attacin and diptericin (an anti-Gram-negative bacterial peptide) are known to be involved in the Imd pathway, and cecropin and defensin are common AMPs in insects and involved in $B$. dorsalis immunity [35]. The present study on $B$. dorsalis demonstrated the important immune role of BdPGRP-SA in recognizing DAP-PGN and activating the production of AMPs against pathogen infection. High expression of all four AMPs was induced by Gram-negative E. coli and three of them were inhibited by dsBdPGRP-SD. However, no changes in AMPs expression occurred when BdPGRP-SA was silenced. BdPGRP-SD also plays an important role in response to 
Gram-negative bacteria infection. In a previous study, diptericin was induced via Gram-negative bacteria in B. dorsalis [36], possibly because of recognition by BdPGRP-SD. The induction by PGN-SA and inhibition of cecropin and defensin by exogenous dsBdPGRP-SD indicates its role in recognition of Gram-positive bacteria. Similarly, the PGRP-SD mutant is more sensitive to S. aureus in Drosophila [20].

\section{Conclusions}

We cloned two PGRP genes, BdPGRP-SA and BdPGRP-SD in B. dorsalis, and determined their roles in response to Gram-negative and Gram-positive pathogen infection. Both genes were highly expressed in adult fat body. Their inducible expressions by PGN indicated potential functions and these were validated using RNAi analysis and AMPs gene expression. In the innate immune system of B. dorsalis, BdPGRP-SA plays an essential role in response against Gram-positive pathogens, and BdPGRP-SD is important in response to Gram-negative pathogens, as well as Gram-positive pathogens. They act with important roles, in different ways, by regulating downstream AMPs gene expression.

Supplementary Materials: The following are available online at http:/ /www.mdpi.com/2075-4450/10/3/79/s1, Table S1: Primer sequence used for cloning and quantitative real-time PCR, Table S2: Amino sequences of PGRPs used in the phylogenetic tree.

Author Contributions: D.W. and J.-J.W. conceived and designed the experiments; Y.-W.L. and Y.-X.Z. contributed materials and performed the experiments; D.W. and Y.-W.L. analyzed the data; D.W., Y.-W.L., and J.-J.W. wrote the paper.

Funding: This work was supported in part by PhD Research Funding of Southwest University (SWU118110), the earmarked fund for the Modern Agro-industry (Citrus) Technology Research System of China (CARS-27), and the 111 Project (B18044).

Conflicts of Interest: The authors declare no conflict of interest. The funders had no role in the design of the study; in the collection, analyses, or interpretation of data; in the writing of the manuscript, or in the decision to publish the results.

\section{References}

1. Hoffmann, J.A.; Reichhart, J.M. Drosophila innate immunity: An evolutionary perspective. Nat. Immunol. 2002, 3, 121-126. [CrossRef] [PubMed]

2. Hultmark, D. Drosophila immunity: Paths and patterns. Curr. Opin. Immunol. 2003, 15, 12-19. [CrossRef]

3. Kimbrell, D.A.; Beutler, B. The evolution and genetics of innate immunity. Nat. Rev. Genet. 2001, 2, $256-267$. [CrossRef]

4. Lemaitre, B. The road to Toll. Nat. Rev. Immunol. 2004, 4, 521-527. [CrossRef] [PubMed]

5. Lemaitre, B.; Hoffmann, J. The host defense of Drosophila melanogaster. Annu. Rev. Immunol. 2007, 25, 697-743. [CrossRef]

6. Janeway, C.A.J. Approaching the asymptote? Evolution and revolution in immunology. J. Immunol. 1989, 54, 1-13. [CrossRef]

7. Leulier, F.; Parquet, C.; Pili-Floury, S.; Ryu, J.-H.; Caroff, M.; Lee, W.-J.; Mengin-Lecreulx, D.; Lemaitre, B. The Drosophila immune system detects bacteria through specific peptidoglycan recognition. Nat. Immunol. 2003, 4, 478-484.

8. Kaneko, T.; Goldman, W.E.; Mellroth, P.; Steiner, H.; Fukase, K.; Kusumoto, S.; Harley, W.; Fox, A.; Golenbock, D.; Silverman, N. Monomeric and polymeric Gram-negative peptidoglycan but not purified LPS stimulate the Drosophila IMD pathway. Immunity 2004, 20, 637-649. [CrossRef]

9. Stenbak, C.R.; Ryu, J.H.; Leulier, F.; Pilifloury, S.; Parquet, C.; Hervé, M.; Chaput, C.; Boneca, I.G.; Lee, W.J.; Lemaitre, B. Peptidoglycan molecular requirements allowing detection by the Drosophila immune deficiency pathway. J. Immunol. 2004, 173, 7339-7348. [CrossRef]

10. MyllymKi, H.; Valanne, S.; Rämet, M. The Drosophila Imd signaling pathway. J. Immunol. 2014, $192,3455$. [CrossRef]

11. Gobert, V.; Gottar, M.; Matskevich, A.A.; Rutschmann, S.; Royet, J.; Belvin, M.; Hoffmann, J.A.; Ferrandon, D. Dual activation of the Drosophila toll pathway by two pattern recognition receptors. Science 2003, 302, 2126-2130. [CrossRef] [PubMed] 
12. Valanne, S.; Wang, J.H.; Rämet, M. The Drosophila Toll signaling pathway. J. Immunol. 2011, 186, 649-656. [CrossRef] [PubMed]

13. Dziarski, R. Recognition of bacterial peptidoglycan by the innate immune system. Cell. Mol. Life Sci. 2003, 60, 1793-1804. [CrossRef]

14. Werner, T.; Liu, G.; Kang, D.; Ekengren, S.; Steiner, H.; Hultmark, D. A family of peptidoglycan recognition proteins in the fruit fly Drosophila melanogaster. Proc. Natl. Acad. Sci. USA 2000, 97, 13772-13777. [CrossRef] [PubMed]

15. Zhu, X.; Zhang, M.; Yao, F.; Yin, Y.; Zou, X.; Hou, L. Involvement of PGRP-SC2 from Artemia sinica in the innate immune response against bacteria and expression pattern at different developmental stages. Dev. Comp. Immunol. 2016, 67, 276-286. [CrossRef] [PubMed]

16. Mellroth, P.; Karlsson, J.; Steiner, H. A scavenger function for a Drosophila peptidoglycan recognition protein. J. Biol. Chem. 2003, 278, 7059-7064. [CrossRef] [PubMed]

17. Zaidmanrémy, A.; Poidevin, M.; Hervé, M.; Welchman, D.P.; Paredes, J.C.; Fahlander, C.; Steiner, H.; Menginlecreulx, D.; Lemaitre, B. Drosophila immunity: Analysis of PGRP-SB1 expression, enzymatic activity and function. PLOS ONE 2011, 6, e17231.

18. Royet, J.; Gupta, D.; Dziarski, R. Peptidoglycan recognition proteins: Modulators of the microbiome and inflammation. Nat. Rev. Immunol. 2011, 11, 837-851. [CrossRef] [PubMed]

19. Michel, T.; Reichhart, J.M.; Hoffmann, J.A.; Royet, J. Drosophila Toll is activated by Gram-positive bacteria through a circulating peptidoglycan recognition protein. Nature 2001, 414, 756-759. [CrossRef] [PubMed]

20. Bischoff, V.; Vignal, C.; Boneca, I.G.; Michel, T.; Hoffmann, J.A.; Royet, J. Function of the drosophila pattern-recognition receptor PGRP-SD in the detection of Gram-positive bacteria. Nat. Immunol. 2004, 5, 1175-1180. [CrossRef] [PubMed]

21. Tanaka, H.; Sagisaka, A. Involvement of peptidoglycan recognition protein 16 in activation of immune deficiency pathway in the immune responsive silkworm cells. Arch. Insect Biochem. Physiol. 2016, 92, 143-156. [CrossRef] [PubMed]

22. Chen, K.; Lin, Z.; Feng, C.; Peng, Y.; Lu, Z. Peptidoglycan recognition protein-S5 functions as a negative regulator of the antimicrobial peptide pathway in the silkworm, Bombyx mori. Dev. Comp. Immunol. 2016, 61, 126-135. [CrossRef] [PubMed]

23. Wang, L.; Gilbert, R.J.C.; Atilano, M.L.; Filipe, S.R.; Gay, N.J.; Ligoxygakis, P. Peptidoglycan recognition protein-SD provides versatility of receptor formation in Drosophila immunity. Proc. Natl. Acad. Sci. USA 2008, 105, 11881-11886. [CrossRef] [PubMed]

24. Leone, P.; Bischoff, V.; Kellenberger, C.; Hetru, C.; Royet, J.; Roussel, A. Crystal structure of Drosophila PGRP-SD suggests binding to DAP-type but not lysine-type peptidoglycan. Mol. Immunol. 2008, 45, 2521-2530. [CrossRef]

25. Iatsenko, I.; Kondo, S.; Mengin-Lecreulx, D.; Lemaitre, B. PGRP-SD, an extracellular pattern-recognition receptor, enhances peptidoglycan-mediated activation of the Drosophila Imd pathway. Immunity 2016, 45, 1013-1023. [CrossRef]

26. Clarke, A.R.; Armstrong, K.F.; Carmichael, A.E.; Milne, J.R.; Raghu, S.; Roderick, G.K.; Yeates, D.K. Invasive phytophagous pests arising through a recent tropical evolutionary radiation: The Bactrocera dorsalis complex of fruit flies. Annu. Rev. Entomol. 2005, 50, 293-319.

27. Wang, J.J.; Wei, D.; Dou, W.; Hu, F.; Liu, W.F.; Wang, J.J. Toxicities and synergistic effects of several insecticides against the oriental fruit fly (Diptera: Tephritidae). J. Econ. Entomol. 2013, 106, 970-978. [CrossRef] [PubMed]

28. Wei, D.; Feng, Y.C.; Wei, D.D.; Yuan, G.R.; Dou, W.; Wang, J.J. Female remating inhibition and fitness of Bactrocera dorsalis (Diptera: Tephritidae) associated with male accessory glands. Fla. Entomol. 2015, 98, 52-58. [CrossRef]

29. Wei, D.; Jia, H.T.; Zhang, M.Y.; Li, R.; Smagghe, G.; Wang, J.J. Comparative analysis of differential gene expression profiling of sex-bias fat body of Bactrocera dorsalis (Diptera: Tephritidae) identifying a new vitellogenin gene. Ann. Entomol. Soc. Am. 2018, 111, 43-54. [CrossRef]

30. Waterhouse, A.M.; Procter, J.B.; Martin, D.M.; Clamp, M.; Barton, G.J. Jalview Version 2-A multiple sequence alignment editor and analysis workbench. Bioinformatics 2009, 25, 1189-1191. [PubMed]

31. Tamura, K.; Peterson, D.; Peterson, N.; Stecher, G.; Nei, M.; Kumar, S. MEGA5: Molecular evolutionary genetics analysis using maximum likelihood, evolutionary distance, and maximum parsimony methods. Mol. Biol. Evol. 2011, 28, 2731-2739. [CrossRef] 
32. Shen, G.M.; Dou, W.; Huang, Y.; Jiang, X.Z.; Smagghe, G.; Wang, J.J. In silico cloning and annotation of genes involved in the digestion, detoxification and RNA interference mechanism in the midgut of Bactrocera dorsalis [Hendel (Diptera: Tephritidae)]. Insect Mol. Biol. 2013, 22, 354-365. [CrossRef]

33. Livak, K.J.; Schmittgen, T.D. Analysis of relative gene expression data using real-time quantitative PCR and the $2^{-\Delta \Delta \mathrm{Ct}}$ method. Methods 2001, 25, 402-408.

34. Zhang, M.Y.; Wei, D.; Li, R.; Jia, H.T.; Liu, Y.W.; Taning, C.N.T.; Wang, J.J.; Smagghe, G. Cytoplasmic glutamine synthetase gene expression regulates larval development in Bactrocera dorsalis (Hendel). Arch. Insect Biochem. Physiol. 2018, 97, e21447.

35. Liu, S.H.; Wei, D.; Yuan, G.R.; Jiang, H.B.; Dou, W.; Wang, J.J. Antimicrobial peptide gene cecropin-2 and defensin respond to peptidoglycan infection in the female adult of oriental fruit fly, Bactrocera dorsalis (Hendel). Comp. Biochem. Physiol. B 2017, 206, 1-7. [CrossRef]

36. Jiang, X.Z.; Cong, L.; Niu, J.Z.; Dou, W.; Wang, J.J. Alternative splicing contributes to the coordinated regulation of ferritin subunit levels in Bactrocera dorsalis (Hendel). Sci. Rep. 2014, 4, 4806. [CrossRef] [PubMed]

37. Shi, Y.; Wang, L.; Dou, W.; Jiang, H.B.; Wei, D.D.; Wei, D.; Niu, J.Z.; Wang, J.J. Determination of instars of Bactrocera dorsalis (Diptera: Tephritidae). Fla. Entomol. 2017, 100, 270-275.

38. Royet, J.; Reichhart, J.M.; Hoffmann, J.A. Sensing and signaling during infection in Drosophila. Curr. Opin. Immunol. 2005, 17, 11-17. [CrossRef]

39. Guan, R.; Mariuzza, R.A. Peptidoglycan recognition proteins of the innate immune system. Trends Microbiol. 2007, 15, 127-134. [CrossRef] [PubMed]

40. Zhao, S.; Wang, X.; Cai, S.; Zhang, S.; Luo, H.; Wu, C.; Zhang, R.; Zhang, J. A novel peptidoglycan recognition protein involved in the prophenoloxidase activation system and antimicrobial peptide production in Antheraea pernyi. Dev. Comp. Immunol. 2018, 86, 78-85.

41. Wang, S.; Beerntsen, B.T. Functional implications of the peptidoglycan recognition proteins in the immunity of the yellow fever mosquito, Aedes aegypti. Insect Mol. Biol. 2015, 24, 293-310. [CrossRef]

42. Wang, J.; Song, X.; Wang, M. Peptidoglycan recognition proteins in hematophagous arthropods. Dev. Comp. Immunol. 2018, 83, 89-95. [CrossRef]

43. Dimopoulos, G.; Christophides, G.K.; Meister, S.; Schultz, J.; White, K.P.; Barillas-Mury, C.; Kafatos, F.C. Genome expression analysis of Anopheles gambiae: Responses to injury, bacterial challenge, and malaria infection. Proc. Natl. Acad. Sci. USA 2002, 99, 8814-8819. [CrossRef] [PubMed]

44. Kurata, S.; Ariki, S.; Kawabata, S. Recognition of pathogens and activation of immune responses in Drosophila and horseshoe crab innate immunity. Immunobiology 2006, 211, 237-249. [CrossRef] [PubMed]

45. Wang, S.; Beerntsen, B.T. Insights into the different functions of multiple peptidoglycan recognition proteins in the immune response against bacteria in the mosquito, Armigeres subalbatus. Insect Biochem. Mol. Biol. 2013, 43, 533-543. [CrossRef]

46. Wang, R.J.; Lin, Z.; Jiang, H.; Li, J.C.; Saha, T.T.; Lu, Z.Y.; Lu, Z.Q.; Zou, Z. Comparative analysis of peptidoglycan recognition proteins in endoparasitoid wasp Microplitis mediator. Insect Sci. 2017, 24, 2-16. [CrossRef] [PubMed]

47. Wei, D.; Tian, C.B.; Liu, S.H.; Wang, T.; Smagghe, G.; Jia, F.X.; Dou, W.; Wang, J.J. Transcriptome analysis to identify genes for peptides and proteins involved in immunity and reproduction from male accessory glands and ejaculatory duct of Bactrocera dorsalis. Peptides 2016, 80, 48-60. [CrossRef] [PubMed]

48. Pili-Floury, S.; Leulier, F.; Takahashi, K.; Saigo, K.; Samain, E.; Ueda, R.; Lemaitre, B. In vivo RNA interference analysis reveals an unexpected role for GNBP1 in the defense against Gram-positive bacterial infection in Drosophila adults. J. Biol. Chem. 2004, 279, 12848-12853.

(C) 2019 by the authors. Licensee MDPI, Basel, Switzerland. This article is an open access article distributed under the terms and conditions of the Creative Commons Attribution (CC BY) license (http:/ / creativecommons.org/licenses/by/4.0/). 